\title{
MicroRNA-214 Prevents Pulmonary Angiogenesis and Alveolarization in Rat Models with Bronchopulmonary Dysplasia via PIGF-Dependent STAT3 Signaling Pathway
}

\section{Zhi-Qun Zhang ( $\nabla$ zhiqun.zhang@zju.edu.cn )}

Affilated Hangzhou First People's Hospital, Zhejiang University School of Medicine https://orcid.org/0000-0002-9068-9715

Hui Hong

Affilated Hangzhou First People's Hospital, Zhejiang University School of Medicine Jing Li

Affilated Hangzhou First People's Hospital, Zhejiang University School of Medicine Xiao-Xia Li

Affilated Hangzhou First People's Hospital, Zhejiang University School of Medicine

\section{Xian-Mei Huang}

Affiliated Hangzhou First People's Hospital, Zhejiang University School of Medicine

\section{Research}

Keywords: MicroRNA-214, PIGF, STAT3 signaling pathway, Bronchopulmonary dysplasia, Pulmonary angiogenesis, Alveolarization

Posted Date: July 28th, 2020

DOI: https://doi.org/10.21203/rs.3.rs-48068/v1

License: (c) (i) This work is licensed under a Creative Commons Attribution 4.0 International License. Read Full License 


\section{Abstract}

Background: In recent years, the roles of microRNAs (miRNAs) in pulmonary diseases have been widely studied and researched. However, the molecular mechanism by which miR-214 affects bronchopulmonary dysplasia (BPD) remains elusive and merits further exploration. Hence, this study aims to clarify the function of miR-214 in pulmonary angiogenesis and alveolarization in preterm infants with BPD.

Methods: BPD neonatal rat model was induced by hyperoxia, and pulmonary epithelial cells were isolated from rats and exposed to hyperoxia. Gain- or loss-of-function experiments were performed in BPD neonatal rats and hyperoxic pulmonary epithelial cells. MiR-214 and PIGF expression in BPD neonatal rats, and eNOS, Bcl-2, c-myc, Survivin, a-SMA and E-cadherin expression in hyperoxic pulmonary epithelial cells were detected using RT-qPCR and western blot analysis. The interaction between PIGF and miR-214 was identified using dual luciferase reporter gene assay and RIP assay. ELISA was adopted to assess IL$1 \beta$, TNF-a, IL-6, ICAM-1 and Flt-1 expression in rats.

Results: Decreased miR-214 expression and elevated PIGF expression were evident in the lung tissues of neonatal rats with BPD. PIGF was a target of miR-214, and miR-214 downregulated PIGF to inactivate the STAT3 signaling pathway. miR-214 overexpression or PIGF silencing decreased apoptosis of hyperoxic pulmonary epithelial cells and declined pulmonary angiogenesis and alveolarization in BPD neonatal rats.

Conclusions: Collectively, miR-214 can protects against pulmonary angiogenesis and alveolarization in preterm infants with BPD by suppressing PIGF and blocking STAT3 signaling pathway.

\section{Introduction}

As a chronic lung disease, bronchopulmonary dysplasia (BPD) afflicts preterm infants and is characterized by retarded lung growth. It remains a principle cause of neonatal morbidity and triggers serious adverse consequences [1, 2]. Around $45 \%$ of the preterm infants whose gestation was below 29 weeks are found to have BPD [3]. It has already been documented that hyperoxia-induced acute lung injury is a key promoter of BPD pathogenesis in preterm infants [4]. It is well known that lung alveolarization could cause incapacity of lungs to exchange gas effectively [5]. However, the underlying molecular mechanisms are still largely unknown.

Through mediation of cellular proliferation, differentiation and metastasis, microRNAs (miRNAs or miRs) are involved in the pathogenesis of multiple diseases and thus have great potentials in serving as therapeutic targets [6]. MiR-214, as a pivotal oncomiR, is upregulated in various kinds of diseases and cancers [7]. In lung cancer, miR-214-3p has been demonstrated to downregulate fibroblast growth factor receptor 1 and to provide beneficial effects to patients [8]. Also, downregulation of miR-214 could reverse the erlotinib resistance in non-small-cell lung cancer by upregulating its direct target gene LHX6 [9]. In our study, placental growth factor (PIGF) was predicted to the target gene of miR-214. Moreover, it has been 
predicted in a prior study that PIGF might serve as a potential biomarker for BPD occurrence [10]. Besides, PIGF could be increased under hyperoxic exposure and downregulating PIGF ameliorated the hyperoxiainduced lung impairment in neonatal rats [11]. PIGF has also been proved to accelerate the phosphorylation of STAT3 [12]. Moreover, neonatal exposure to hyperoxia has been found to lead to a significant increase of the signal transducer and activator of transcription 3 (STAT3) mRNA expression in pulmonary endothelial cells [13]. In this regard, we hypothesized that a regulatory network of miR214/PIGF/STAT3 signaling pathway may be involved in BPD. Therefore, the current study was conducted with the aim to verify the expected involvement of miR-214/PIGF/STAT3 axis in BPD, and to elucidate the underlying molecular mechanisms.

\section{Materials And Methods}

\subsection{Compliance with ethical standards.}

Animal experiment protocols were approved by the Experimental Animal Ethics Committee of Affiliated Hangzhou First People's Hospital, Zhejiang University School of Medicine. All animal experiments were performed in accordance with the Guide for the Care and Use of Laboratory animals published by the US National Institutes of Health. Extensive efforts were made to ensure minimal suffering of animals during the study.

2.2. Analysis of BPD gene expression dataset and bioinformatics prediction.

The gene expression dataset GSE25293 of mouse BPD models was retrieved from the annotation platform GPL1261 in the Gene Expression Omnibus (GEO) database (https://www.ncbi.nlm.nih.gov/gds) and then analyzed using R language. The databases DIANA TOOLS (http://diana.imis.athenainnovation.gr/DianaTools/), miRWalk (energy <-30) (http://mirwalk.umm.uni-heidelberg.de), mirDIP (Integrated Score > 0.6) (http://ophid.utoronto.ca/mirDIP/), miRDB (http://www.mirdb.org) and miRSearch (https://www.exiqon.com/miRSearch) were used to analyze the intersected upstream miRNAs in human body. A box plot was drawn using $\mathrm{R}$ language to extract key miRNA expression data from the miRNA dataset GSE25293 from the annotation platform GPL11199 in the GEO database. Protein-protein interaction (PPI) analysis was performed on String website (https://string-db.org) to obtain proteins that could potentially bind to BPD. Cytoscape (https://cytoscape.org) was used to process the visual graphs of PPI analysis, and its downstream regulatory pathways were predicted based on existing literatures.

\subsection{Establishment of hyperoxia-induced BPD rat models.}

Twelve specific-pathogen-free (SPF) Sprague-Dawley (SD) rats (a gestational age of 15 days; Shanghai SLAC Laboratory Animal Co., Ltd., Shanghai, China) were raised at $22 \pm 3{ }^{\circ} \mathrm{C}$ and with humidity of $60 \pm 5 \%$ and circadian rhythm of $12 \mathrm{~h}$. Each neonatal rat was housed individually and was self-delivered after 1 week of acclimation. Then 60 neonatal rats within $12 \mathrm{~h}$ of birth difference after delivery were randomly grouped into the hyperoxia treatment group and the control group. And the hyperoxia-treated rats with BPD were then treated with miR-214 negative control (NC), miR-214 agomir, miR-214 NC + PIGF vector, 
and miR-214 agomir + PIGF ( $\mathrm{n}=12$ in each treatment). After the recombinant adenoviruses were packaged, cloned, amplified, purified and titrated, the hyperoxia-induced BPD model rats were developed as previously reported $[14,15]$. The remaining experimental control conditions and operations were the same as those in the hyperoxia treatment group. The box was routinely opened for 30 min every day, and water and food were added and the litter was replaced. The mother rats were exchanged with the control group (to avoid the decreased feeding ability of mother rats because of oxygen toxicity). The rats in control group were placed in the same room, with the similar experimental control factors to those in the hyperoxia treatment group. Three newborn rats were randomly selected from the two groups by random number method at the 3rd, 7th and 14th days after the experiment began, and then received intraperitoneal injection of $90 \mathrm{mg} / \mathrm{kg}$ pentobarbital sodium for anesthesia. Next, the abdominal cavity was opened immediately, and the right lungs were taken out and placed in an RNase-free cryo vial (Eppendorf, Hamburg, Germany). After rapidly frozen with liquid nitrogen, the lungs were stored in a $-80^{\circ} \mathrm{C}$ refrigerator for subsequent reverse transcription quantitative polymerase chain reaction (RT-qPCR) and western blot analysis. Then $40 \mathrm{~g} / \mathrm{L}$ paraformaldehyde was slowly injected to the rats through the left bronchus until the apex of lung was inflated, placed in an embedding box, and added with $40 \mathrm{~g} / \mathrm{L}$ paraformaldehyde solution for overnight fixation for subsequent detection.

\subsection{Enzyme-linked immunosorbent (ELISA) assay.}

The strips used in the experiment were equilibrated at room temperature for $20 \mathrm{~min}$. Standard and sample

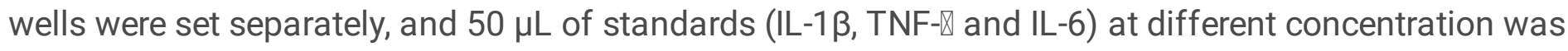
added into the standard wells respectively. Then $10 \mu \mathrm{L}$ samples and were supplemented into the sample wells and $40 \mu \mathrm{L}$ sample dilution was then added to the samples. The blank wells were not subject to treatment. Next, $100 \mu \mathrm{L}$ of horse radish peroxidase (HRP)-labeled antibody to be detected was added to the standard wells and the sample wells respectively, and the blank wells were not subject to treatment. The wells were sealed with microplate sealers, followed by 60 -min incubation at $37^{\circ} \mathrm{C}$. After the liquid was discarded, the experimental strips were washed in full-automatic washing machine. Then $50 \mu \mathrm{L}$ of substrate $A$ and $B$ were added to each well, followed by 15 -min incubation at $37^{\circ} \mathrm{C}$ in subdued light. Subsequently, $50 \mu \mathrm{L}$ of the stop buffer was added to each well and allowed to stand for $15 \mathrm{~min}$, after which the OD value of each well was measured at a wavelength of $450 \mathrm{~nm}$.

\subsection{Hematoxylin-eosin (HE) staining.}

The lung tissues of rats in each group were fixed with $4 \%$ paraformaldehyde for $24 \mathrm{~h}$, dehydrated with $80 \%, 90 \%$ and $100 \%$ ethanol and $n$-butanol respectively, and immersed in a wax box at $60{ }^{\circ} \mathrm{C}$. Following xylene dewaxing and hydration, the sections were first stained with hematoxylin (Beijing Solarbio Science \& Technology Co., Ltd., Beijing, China) for 2 min, stained with eosin for 1 min, followed by gradient ethanol dehydration, xylene clearing, and neutral rubber fixing. Finally, the morphological changes of lung tissues were observed and analyzed under an optical microscope (XP-330, Bingyu Optical Instrument Co., Ltd., Shanghai, China).

2.6. Alveolar epithelial cell isolation using Immunomagnetic bead. 
The fetal rats were taken out by cesarean section from the SD rats with a gestational age of 15 days under sterile condition and were transected at the chest, after which the lungs were taken out and put in pre-cooled phosphate buffer solution (PBS) to remove residual non-lung tissues. Digestion, filtration, centrifugation, and sorting were carried out according to the experimental operations. CD14 magnetic beads were added to the cells $\left(20 \mu \mathrm{L} / 10^{7}\right.$ cells), mixed well, followed by 15 -min culture at $4-8{ }^{\circ} \mathrm{C}$. The cells were washed with buffer solution ( $1 \mathrm{~mL} / 10^{7}$ cells) and centrifuged at $1500 \mathrm{r} / \mathrm{min}$ for $10 \mathrm{~min}$, with the supernatant completely removed. The cells were resuspended in $500 \mu \mathrm{L}$ of buffer solution, and the cell suspension was added to a MS separation column. The unlabeled cells that had flowed out first were collected, which were negative cells. The MS separation column was washed with $1500 \mu \mathrm{L}$ of buffer solution. The separation column was removed from the magnetic field and the cells retained on the column were quickly eluted with $1 \mathrm{~mL}$ of buffer solution. These cells were magnetically labeled positive cells. Under a modified Barthel microscope, more dark particles in the cytoplasm were visible with characteristic eosinophils observed, which presented with obvious microvilli in lamellar bodies and cell membranes. This indicated that type II alveolar epithelial cells in the fetal rats were successfully isolated.

\subsection{Development of hyperoxia-provoked cell injury models.}

Pulmonary epithelial cells after 2 days of growth were exposed to air (control) or hyperoxia. The cells exposed to air and hyperoxia were placed in a closed oxygen chamber with $21 \%$ oxygen volume fraction. The hyperoxic cells were further transfected with plasmids of miR-214 NC, miR-214 mimic, miR-214 NC + PIGF vector, miR-214 NC + PIGF, and miR-214 mimic + PIGF. Then the recombinant adenoviruses were packaged, cloned, amplified, purified and titrated. The pulmonary epithelial cells were infected with these adonoviruses and placed in a closed oxygen chamber with $85 \%$ oxygen volume fraction.

\subsection{Transmission electron microscope.}

After the pulmonary epithelial cells were centrifuged at 10,000 rpm for $10 \mathrm{~min}$, the supernatant was discarded and the cells were fixed in $4 \%$ glutaraldehyde at $4{ }^{\circ} \mathrm{C}$ for more than $2 \mathrm{~h}$. The cells were fixed with $1 \%$ osmium tetroxide for $2 \mathrm{~h}$, and dehydrated with gradient ethanol and acetone. The cells were then immersed with epoxy resin, embedded and polymerized, and then made into semi-thin sections with a thickness of $0.5 \mu \mathrm{m}$. The sections were positioned under a microscope, stained with uranyl acetate and lead citrate, and observed and photographed under a transmission electron microscope $(\mathrm{H}-7500)$.

\subsection{RNA binding protein immunoprecipitation (RIP).}

The pulmonary epithelial cells were added with $10 \mathrm{~mL}$ of PBS, scraped off with a cell scraper and transferred into a centrifuge tube. The cells were centrifuged at $1500 \mathrm{rpm}$ for $5 \mathrm{~min}$ at $4{ }^{\circ} \mathrm{C}$, then added with RIP Lysis Buffer, mechanically dissociated into and mixed thoroughly, and lysed on ice for 5 min to prepare cell lysate. Next, $50 \mu \mathrm{L}$ of magnetic beads were added into each tube and mixed well, after which $0.5 \mathrm{~mL}$ RIP Wash Buffer was added to rinse the magnetic beads, and $100 \mu \mathrm{L}$ RIP Wash Buffer was added to resuspend beads. Then $5 \mu \mathrm{g}$ of Ago 2 antibody was added to the tubes and incubated in rotation for $30 \mathrm{~min}$ at room temperature. The supernatant was discarded and the beads were washed twice with 
$0.5 \mathrm{~mL}$ RIP Wash Buffer for subsequent experiments. $900 \mu \mathrm{L}$ of RIP Immunoprecipitation Buffer was added to the magnetic bead-antibody mixture, after which the centrifugation was carried out at $14000 \mathrm{rpm}$ for $10 \mathrm{~min}$ at $4{ }^{\circ} \mathrm{C}$. The supernatant was collected and transferred into a new eppendorf (EP) tube, and then $100 \mu \mathrm{L}$ of the supernatant was taken into the tube containing the magnetic bead-antibody. $1.0 \mathrm{~mL}$ served as the final volume of the immunoprecipitation reaction, and incubation was conducted overnight at $4{ }^{\circ} \mathrm{C}$. Then the magnetic beads were washed 6 times with $0.5 \mathrm{~mL}$ RIP Wash Buffer, $150 \mu \mathrm{L}$ of proteinase $\mathrm{K}$ buffer was added, and the RNA was purified by 30 min of incubation at $55^{\circ} \mathrm{C}$. The RNA was extracted by a conventional TRIzol method followed by RT-qPCR detection.

\subsection{Giemsa staining.}

The rats were anesthetized by intraperitoneal injection of $1 \%$ sodium pentobarbital solution, and fixed on a simple operation table. The thoracic cavities of rats were exposed after being euthanized by exsanguination, after which the pleural tissues around the trachea were bluntly separated, the trachea was fully exposed and a needle was used to stab at the $1 / 3$ of the trachea. The tip of the $18 \mathrm{G}$ indwelling needle was previously trimmed into a hernia type, and the tracheal intubation was performed along the trial point and ligated with a surgical line. $1 \mathrm{~mL}$ of pre-cooled sterile normal saline was used to perfuse the lung tissues of the rats for three times. The bronchoalveolar lavage fluid (BALF) was collected and stored in a pre-cooled EP tube and subsequently centrifuged at $1200 \mathrm{r} / \mathrm{min}$ for $20 \mathrm{~min}$ at $4{ }^{\circ} \mathrm{C}$. The supernatant was stored in a $-80^{\circ} \mathrm{C}$ freezer for subsequent use. The cell precipitate was resuspended in $100 \mu \mathrm{L}$ of PBS, smeared with $50 \mu \mathrm{L}$ of the suspension, and stained with Swiss Giemsa. The number of neutrophils was counted under an oil microscope and the total number of cells in $10 \mu \mathrm{L}$ of the suspension was counted by a hemocytometer.

\subsection{Immunohistochemistry.}

The specimen was fixed in 10\% formaldehyde, followed by preparation of $4 \mu \mathrm{m}$ paraffin-embedded sections. Then the tissue sections were placed in a $60^{\circ} \mathrm{C}$ oven for $1 \mathrm{~h}$, dewaxed by xylene in conventional manner, and then dehydrated with gradient alcohol, followed by 30 -min culture in $3 \% \mathrm{H}_{2} \mathrm{O}_{2}$ (SigmaAldrich, Shanghai, China) at $37^{\circ} \mathrm{C}$. Then the tissue sections were boiled in $0.1 \mathrm{M}$ citrate buffer solution for 20 min at $95{ }^{\circ} \mathrm{C}$, and cooled to room temperature. The sections were blocked with $10 \%$ normal goat serum for 10 min at $37^{\circ} \mathrm{C}$ followed by rabbit anti-eNOS (AF0096, Affinity) incubation at $4{ }^{\circ} \mathrm{C}$ for $12 \mathrm{~h}$. The sections were treated with biotin-labeled goat anti-rabbit secondary antibody at room temperature for $10 \mathrm{~min}$. After thoroughly washed, S-A/HRP was added to react at room temperature for $10 \mathrm{~min}$. The tissue sections were developed using diaminobenzidine (DAB) away from light at room temperature for $8 \mathrm{~min}$. Then the tissues were counter-stained with hematoxylin, dehydrated, cleared, blocked, and observed under a light microscope. The number of positive cells was counted using image analysis software (Nikon Corporation, Tokyo, Japan). Five fields of equal area were selected from each section, and the proportion of positive cells was calculated with the average value calculated. The cells with apparent brown or brownish yellow particles in the cytoplasm were positive cells. 
2.12. Dual luciferase reporter gene assay.

The artificially synthesized PIGF 3' untranslated regions (UTR) gene fragment was constructed into pMIRreporter (Promega, Madison, WI, USA). A complementary sequence with mutation of the seed sequence was designed based on the wild type (WT) of PIGF and constructed into the pMIR-reporter reporter plasmid. The correctly sequenced luciferase reporter plasmids WT and MUT were respectively cotransfected with miR-214 mimic and miR-214 NC into HEK293T cells. After $48 \mathrm{~h}$ of transfection, cells were collected and lysed, and the luciferase activity was measured using Dual-Luciferase Reporter Assay System (Promega, Madison, WI, USA).

\subsection{RT-qPCR assay.}

Total RNA was extracted from cells using the Trizol kit (Invitrogen Inc., Carlsbad, CA, USA) and reverse transcribed into cDNA according to the instructions of TaqMan MicroRNA Assays Reverse Transcription Primer (4427975, Applied Bio-systems, Foster City, CA,USA). The reverse transcribed cDNA was diluted to $50 \mathrm{ng} / \mu \mathrm{L}$. The expression of relevant genes was analyzed normalized to U6 (for miRNA) and glyceraldehyde-3-phosphate dehydrogenase (GAPDH) (for other genes). The fold changes were calculated using relative quantification (the $2^{-\triangle \Delta \mathrm{Ct}}$ method). The primers used are shown in Table 1 . 
TABLE 1

Primer sequences of RT-qPCR

\begin{tabular}{|ll|}
\hline Gene & Primer sequences \\
\hline GAPDH & F: TCTGATTTGGTCGTATTGGG \\
\hline UAPDH & R: GGAAGATGGTGATGGGATT \\
\hline U6 F & F: CTCGCTTCGGCAGCACA \\
\hline hsa-miR-214 & R: AACGCTTCACGAATTTGCGT \\
\hline hsa-miR-214 & R: CACCTTTCTCCCTTTCCCCTTACTCTCC \\
mmu-miR-214 & F: ACACTCCAGCTGGGACAGCAGGCACAGACA \\
\hline mmu-miR-214 & R: TGGTGTCGTGGAGTCG \\
PIGF & F: CGGAATTCCACCATGCCGGTCATGAGGCTGTTCCCT \\
PIGF & R: CCAGATCTTACCTCCGGGGAACAGCATCGCC \\
eNOS & F: AGACGGACCCAAGTTTCCTC \\
\hline Enos & R: TCCCGAGCATCAAATACCTG \\
\hline Bcl-2 & F: TTTCTCCTGGCTGTCTCTGAA \\
\hline Bcl-2 & R: TGTGTGTGTGTGTGTTCTGCT \\
\hline C-myc & F: ACAGCGTCTGCTCCACCT \\
\hline C-myc & R: CTGCGTAGTTGTGCTGATGT \\
\hline $\begin{array}{l}\text { Note: GAPDH, glyceraldehyde-3-phosphate dehydrogenase; PIGF, placental growth factor (PIGF); } \\
\text { eNOS, endothelial nitric-oxide synthase; F, forward; R, reverse. }\end{array}$ \\
\hline
\end{tabular}

\subsection{Western blot assay.}

The lung tissues or cells were added with lysis buffer, shaken on a vortex agitator, and centrifuged at $12000 \mathrm{r} / \mathrm{min}$ for $30 \mathrm{~min}$ at $4{ }^{\circ} \mathrm{C}$ to remove tissues or cell debris. The supernatant was taken and the total protein concentration was measured using a bicinchoninic acid (BCA) kit. $50 \mu \mathrm{g}$ of protein was subjected to $10 \%$ sodium dodecyl sulfate polyacrylamide gel electropheresis and electroblotted to polyvinylidene fluoride membranes by wet transfer method. After being blocked with $5 \%$ skim milk powder at room temperature for $1 \mathrm{~h}$, the membrane was then probed with diluted primary antibodies from CST (Danvers, MA, USA) against Survivin (\# 2808S), GAPDH (\#5174), B-cell lymphoma-2 (Bcl-2, \#3498) and c-myc (\#13987), and primary antibodies from Abcam (Cambridge, UK) against PIGF (ab74778), E-cadherin (ab11512) and a-smooth muscle actin (a-SMA, ab32575), and then diluted based on the manuals. The membrane was re-probed with HRP-labeled secondary antibody for $1 \mathrm{~h}$. The membrane was placed on a clean glass plate. The immunocomplexes on the membrane were visualized using enhanced 
chemiluminescence (ECL) fluorescence detection kit (BB-3501, Amersham, Little Chalfont, UK), and band intensities were quantified using a Bio-Rad image analysis system and Quantity One v4.6.2 software. The ratio of the gray value of the target band to GAPDH was representative of the relative protein expression.

\subsection{Statistical analysis.}

Data analyses were conducted using SPSS 21.0 (IBM Corp, Armonk, NY, USA). Measurement data were described using mean \pm standard deviation. An unpaired $t$-test was conducted to compare the data obeying normal distribution and homogeneity of variance between two groups. Data comparisons between multiple groups were performed using one-way analysis of variance (ANOVA), followed by a Tukey's multiple comparisons posttest. Data comparisons at different time points were performed by repeated measures ANOVA, followed by a Bonferroni post hoc test for multiple comparisons. Pearson correlation was used to analyze the relationship between two indicators. A value of $p<0.05$ was considered to be statistically significant.

\section{Results}

3.1. miRNA and mRNA expression profiles in BPD.

There has been literature showing that PIGF is an important gene participating in BPD in preterm infants, but the regulatory pathway of this gene is still unknown and possesses great research potential [16]. A boxplot shown in Fig. 1A illustrating that PIGF was highly expressed in BPD model. The predicted results from DIANA TOOLS, miRWalk, mirDIP, miRDB and miRSearch databases revealed that the upstream miRNA number of PIGF (actually PGF was used during prediction) were 80, 196, 5, 58 and 39 respectively. Only one intersected miRNA was obtained shown by the Venn map, which was miR-214 (Fig. 1B). Besides, the binding site suggested by DIANA Tools also indicated the potential interaction between PIGF and miR-214-3p (Fig. 1C). The boxplot on miR-214 expression drawn using R language showed that miR214 was downregulated in BPD neonatal rats induced by hyperoxia (Fig. 1D). Existing studies have shown that miR-214-3p is a miRNA related to lung cancer and is downregulated in lung tumor tissues [17]. PPI analysis indicated that PIGF (PGF) may be related with several signaling pathways (Fig. 1E), and some literature has shown that high expression of PIGF can promote the expression of STAT3 $[18,19]$. Therefore, we hypothesized that miR-214 can regulate the expression of PIGF and further regulate the STAT3 signaling pathway, thus affecting the progression of BPD in preterm infants.

3.2. miR-214 is downregulated in the lung tissues of hyperoxia-induced BPD neonatal rats.

In order to verify miR-214 expression in the lung tissues of neonatal rats exposed to hyperoxia, neonatal rats were selected for experiments. After BPD models were established, RT-qPCR showed that on 3rd, 7th, and 14th days, miR-214 expression in lung tissue of neonatal rats with BPD was lower than that of normal neonatal rats $(p<0.05$; Fig. 2A). RT-qPCR and western blot analysis showed that PIGF expression in the lung tissue of neonatal rats with BPD were higher than that in normal neonatal rats on 3rd, 7th, and 14 th days $(p<0.05$; Fig. 2B-C). Correlation analysis showed a negative correlation between miR-214 and 
PIGF expressed in lung tissues ( $p<0.05$; Fig. 2D). The above results suggested that expression level of miR-214 was decreased and PIGF was elevated in lung tissue of neonatal rats exposed to hyperoxia, and there was a certain correlation between them.

3.3. Overexpression of miR-214 blocks pulmonary angiogenesis and alveolarization in neonatal rats with BPD in vivo.

To validate the effect of miR-214 overexpression on neonatal rats with BPD, we established hyperoxiainduced neonatal rats with BPD and we injected them with miR-214 NC and miR-214 agomir. When compared with neonatal rats exposed to air, the BPD neonatal rats exhibited increased levels of inflammatory factors (IL-1 $\beta$, TNF- $\bigotimes$, IL-6, FIt-1 and ICAM-1) (Fig. 3A-D), elevated eNOS expression (Fig. 3E), and increased number of macrophages (Fig. 3F) (all $p<0.05$ ), which was reversed by miR-214 agomir treatment $(p<0.05)$. HE staining revealed that compared with neonatal rats exposed to air, BPD neonatal rats showed reduced alveoli number and simplified structure, the alveolar wall ruptured and merged into pulmonary bullae, the pulmonary microvessel density was decreased, and the ratio of alveolar area/pulmonary septal area was increased $(p<0.05)$. While after miR-214 treatment, the alveolar structure of BPD neonatal rats exhibited a contrasting trend in the aforementioned factors $(p<0.05$; Fig. 3G-H). The above results indicated that overexpression of miR-214 can prevent pulmonary angiogenesis and alveolarization in neonatal rats with BPD.

3.4. Overexpression of miR-214 decreased the pulmonary epithelial cell apoptosis in vitro.

To elucidate the effect of miR-214 on pulmonary epithelial cells, pulmonary epithelial cells were obtained from rats. Under a transmission electron microscope, compared with pulmonary epithelial cells exposed to air, the pulmonary epithelial cells treated with hyperoxia displayed destroyed cytoplasmic lamellar structure of the alveolar epithelium, with relatively large vacuoles formed and gap between blood-air barriers increased, which can be rescued by miR-214 mimic transfection (Fig. 4A). Next, Western blot analysis result in Fig. 4B-C manifested that in hyperoxic pulmonary epithelial cells, Survivin and Bcl-2 expression was decreased and c-myc expression was increased, while miR-214 mimic treatment triggered an opposite trend. a-SMA expression was increased while E-cadherin expression was decreased in hyperoxic pulmonary epithelial cells, which was annulled miR-214 mimic (Fig. 4D-E). Taken together, overexpression of miR-214 can attenuate pulmonary epithelial cell alteration in BPD rats.

\subsection{PIGF is a target gene of miR-214.}

Then, the regulatory mechanism of miR-214 was explored. Bioinformatics analysis revealed a complementary sequence of PIGF within the sequence of miR-214 (Fig. 5A). Dual luciferase reporter gene assay verified that co-transfection of miR-214 mimic with the 3'-UTR of WT-PIGF showed reduced luciferase activity, whereas the 3'-UTR of MUT-PIGF showed no significant difference (Fig. 5B). RT-qPCR and western blot analysis presented that the mRNA and protein level of PIGF were reduced in cells treated with miR-214 mimic (Fig. 5C-D). RIP experiment further confirmed that the enrichment of miR-214 and 
PIGF was higher in Ago2 group than that in IgG (Fig. 5E). Collectively, miR-214 targeted and regulated the expression of PIGF.

3.6. Overexpressed miR-214 disrupts pulmonary angiogenesis and alveolarization by inactivating PIGFdependent STAT3 signaling pathway in neonatal rats with BPD.

The downstream regulatory pathway of PIGF was predicted using KEGG and GeneMANIA databases and the results showed that PIGF was closely related to the STAT3 signaling pathway (Fig. 6A). Western blot analysis showed that STAT3 phosphorylation was increased in the lung tissue of neonatal rats exposed in hyperoxia (Fig. 6B). The neonatal rats with BPD were then respectively treated with miR-214 NC + PIGF vector, miR-214 NC + PIGF, or miR-214 agomir + PIGF. The results showed that PIGF overexpression

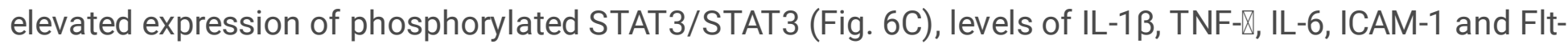
1 (Fig. 6D-E), eNOS expression (Fig. 6F) and number of macrophages (Fig. 6G), which was abrogated by treatment of miR-214 agomir + PIGF (all $p<0.05$ ). Moreover, PIGF upregulation reduced number of alveoli and simplified structure. The alveolar wall ruptured to form a large pulmonary vesicle with declined pulmonary microvessels density and elevated ratio of alveolar area/pulmonary septal area $(A / S)(p<$ 0.05; Fig. $6 \mathrm{H}-\mathrm{I})$, and we observed an opposite trend in alveolar alterations in BPD rats treated with miR214 agomir + PIGF. These results suggested that miR-214 targeted PIGF to inhibit the STAT3 signaling pathway, thus blocking pulmonary angiogenesis and alveolarization in neonatal with BPD.

3.7. Overexpressed miR-214 reduces pulmonary epithelial cell apoptosis via inactivation of PIGFdependent STAT3 signaling pathway.in vitro.

To study the effect of miR-214 on pulmonary epithelial cells of rats with BPD, pulmonary epithelial cells were obtained from rats. Under a transmission electron microscope, in the PIGF-overexpressing rats, the cytoplasmic lamellar structure of the alveolar epithelium was destroyed, large vacuoles were formed, the blood-air barriers gap was increased (Fig. 7A). Moreover, there was a reduction in the expression of antiapoptotic proteins Survivin and Bcl-2 (Fig. 7B-C) as well as a-SMA (Fig. 7D-E), while an increase in the expression of pro-apoptotic protein c-myc (Fig. 7B-C) and E-cadherin $(p<0.05$; Fig. 7D-E). These results in the rats were negated by co-transfected with miR-214 mimic and PIGF ( $p<0.05$; Fig. 7A-E). These results indicated that overexpression of miR-214 targeting PIGF can inhibit the effect of STAT3 signaling pathway on rat bronchial embryonic pulmonary epithelial cells.

\section{Discussion}

BPD is a respiratory condition occurring in preterm neonates and can lead to chronic respiratory problems driven by several prenatal and/or postnatal factors [20]. The known risk factors associated with BPD development in preterm neonates include small gestational age, preeclampsia, chorioamnionitis and infiltration of the chorioamnion by neutrophils [21, 22]. The most likely underlying pathogenesis is the constant inflammation in lung, and thus corticosteroid, which has a strong anti-inflammatory effect, has been employed in the treatment of BPD [23]. In order to highlight a novel therapeutic method to prevent and cure the pulmonary angiogenesis and alveolarization in neonatal infants with BPD, we planned the 
current study, and the in vitro and in vivo results demonstrated that miR-214 could inhibit pulmonary angiogenesis and alveolarization in neonatal infants with BPD via PIGF-dependent STAT3 signaling pathway blockade.

Our findings illustrated that miR-214 was downregulated in hyperoxia-induced BPD neonatal rats. MiR214 , a member of microRNA precursors, plays a pivotal role in the pathogenesis of multiple human disorders, including cardiovascular diseases and cancers [24, 25]. The expression of miR-214 increased by TWIST1 promotes the epithelial-to-mesenchymal transition and metastasis in lung adenocarcinoma [26]. Additionally, our study portrayed that PIGF, which was highly expressed in the lung tissues of preterm rats with BPD, was negatively targeted by miR-214 and activated the STAT3 signaling pathway. PIGF was found to be highly expressed in the BPD rats [27]. Moreover, an inverse correlation has been detected in our study between miR-214 and PIGF, and the post-transcriptional miR-214 possesses the ability to modulate the expression of PIGF in lung tissues [28]. On the basis of a prior study, PIGF could increase the phosphorylation of STAT3 [12]. MiR-214 has been proven to downregulate the expression of STAT3 in human cervical and colorectal cancer cells [29].

Moreover, another critical finding of our study was that miR-214 overexpression could downregulate the expression of IL-1 $\beta$, TNF- $\triangle$ and IL- 6 and repress pulmonary angiogenesis and alveolarization in hyperoxiainduced BPD neonatal rats. IL-1 $\beta$ is one of the main mediators of inflammation and plays a causative role in innumerable diseases [30]. In the primary pathological features of BPD, IL-1 $\beta$ contributes to excessive alveolar elastogenesis through the interaction with av $\beta 6$ which serves as an epithelial or a mesenchymal signaling molecule [31]. TNF is a kind of ligand related to systemic inflammation of human bodies [32]. TNF- $\$ overexpression not only increases the release of glutamate but decreases the cell cycling activity of marrow mesenchymal stem cells [33]. IL-6 is a typical proinflammatory cytokine which plays a functional role in a number of physiological inflammatory and immunological processes [34]. It has been supported that there is a close correlation between the dysregulation of IL- 6 with moderate and severe BPD in preterm infants with a small gestational age [35]. PIGF overexpression contributes an exaggerated inflammatory state [36]. MiR-214 can inhibit angiogenesis procession by suppressing Quaking and pro-angiogenic growth factor expression [37]. Through the depletion of PIGF, Kaempferol exerts suppressive effects on angiogenesis of human retinal endothelial cells [38]. Therefore, our studies evidenced that miR-214 overexpression could reduce the expression of pro-inflammatory factors, thus blocked pulmonary angiogenesis and alveolarization as well as inhibit cell apoptosis of lung epithelium in neonatal rats with BPD.

In conclusion, upregulated miR-214 can potentially block the activation of STAT3 pathway by inhibiting its downstream target gene PIGF, ultimately preventing pulmonary angiogenesis and alveolarization in neonatal infants with BPD (Fig. 8). Investigation of miR-214-PIGF-STAT3 pathway in BPD and their functions yields a better understanding of their vivo mechanisms and may have potentially provide important therapeutic implications in the treatment of BPD. However, the molecular mechanism of the miR-214-PIGF-STAT3 pathway in BPD still requires further elucidation with clinical cases involved in the future. 


\section{Abbreviations}

miRNAs, microRNAs; BPD, bronchopulmonary dysplasia; PIGF, placental growth factor; STAT3, signal transducer and activator of transcription 3; SPF, specific-pathogen-free; SD, Sprague-Dawley; NC, negative control; ELISA, Enzyme-linked immunosorbent; HRP, horse radish peroxidase; HE, Hematoxylin-eosin; PBS, phosphate buffer solution; RIP, RNA binding protein immunoprecipitation; EP, eppendorf; BALF, bronchoalveolar lavage fluid; DAB, diaminobenzidine; UTR, untranslated regions; WT, wild type; GAPDH, glyceraldehyde-3-phosphate dehydrogenase; BCA, bicinchoninic acid; ECL, enhanced chemiluminescence; ANOVA, analysis of variance

\section{Declarations}

\section{Authors' contributions}

ZQZ, HH and XMH conceived and designed research; ZQZ and XXL performed experiments; JL and XMH analyzed data; ZQZ and HH interpreted results of experiments; ZQZ and XXL prepared figures; $\mathrm{HH}$ and $\mathrm{XMH}$ drafted manuscript; ZQZ, XXL and JL edited and revised manuscript; ZQZ, XXL, JL, HH and XMH approved final version of manuscript.

\section{Funding}

This work was supported by Natural Science Foundation of Zhejiang Province (Grant Number: LY20H040008, LY15H040004). The funding source play no role in study design, data collection, data analysis, data interpretation, writing of the report, or in the decision to submit the report for publication.

\section{Availability of data and materials}

Data generated and analyzed as part of this study are included in the manuscript or are available upon request from the corresponding author.

\section{Ethics approval and consent to participate}

All animal experiments were conducted in strict accordance with the Guide to the Management and Use of Laboratory Animals issued by the National Institutes of Health. The protocol of animal experiments was approved by the Institutional Animal Care and Use Committee of Affiliated Hangzhou First People's Hospital, Zhejiang University School of Medicine.

\section{Consent for publication}

Not applicable.

\section{Competing interests}

The authors declare that they have no competing interests. 


\section{Acknowledgements}

We acknowledge and appreciate our colleagues for their valuable efforts and comments regarding this paper.

\section{References}

1. Will JP, Hirani D, Thielen F, Klein F, Vohlen C, Dinger K, Dotsch J, Alejandre Alcazar MA. Straindependent effects on lung structure, matrix remodeling, and Stat3/Smad2 signaling in C57BL/6N and C57BL/6J mice after neonatal hyperoxia. Am J Physiol Regul Integr Comp Physiol. 2019;317(1): R169-R81.

2. Isayama T, Lee SK, Yang J, Lee D, Daspal S, Dunn M, Shah PS, Canadian Neonatal N, Canadian Neonatal Follow-Up Network Revisiting the Definition of Bronchopulmonary Dysplasia: Effect of Changing Panoply of Respiratory Support for Preterm Neonates. JAMA Pediatr. 2017;171(3):271-9.

3. Stoll BJ, Hansen NI, Bell EF, Walsh MC, Carlo WA, Shankaran S, Laptook AR, Sanchez PJ, Van Meurs KP, Wyckoff M, Das A, Hale EC, Ball MB, Newman NS, Schibler K, Poindexter BB, Kennedy KA, Cotten CM, Watterberg KL, D'Angio CT, DeMauro SB, Truog WE, Devaskar U, Higgins RD, Eunice Kennedy Shriver National Institute of Child H, Human Development Neonatal Research N. Trends in Care Practices, Morbidity, and Mortality of Extremely Preterm Neonates, 1993-2012. JAMA. 2015;314(10):1039-51.

4. Syed M, Das P, Pawar A, Aghai ZH, Kaskinen A, Zhuang ZW, Ambalavanan N, Pryhuber G, Andersson S, Bhandari Hyperoxia causes miR-34a-mediated injury via angiopoietin-1 in neonatal lungs. Nat Commun. 2017;8(1):1173.

5. Ruiz-Camp J, Quantius J, Lignelli E, Arndt PF, Palumbo F, Nardiello C, Surate Solaligue DE, Sakkas E, Mizikoval, Rodriguez-Castillo JA, Vadasz I, Richardson WD, Ahlbrecht K, Herold S, Seeger W, Morty RE. Targeting miR-34a/Pdgfra interactions partially corrects alveologenesis in experimental bronchopulmonary EMBO Mol Med. 2019;11(3).

6. Du L, Borkowski R, Zhao Z, Ma X, Yu X, Xie XJ, Pertsemlidis A high-throughput screen identifies miRNA inhibitors regulating lung cancer cell survival and response to paclitaxel. RNA Biol. 2013;10(11):1700-13.

7. Yang L, Zhang W, Wang Y, Zou T, Zhang B, Xu Y, Pang T, Hu Q, Chen M, Wang L, Lv Y, Yin K, Liang H, Chen X, Xu G, Zou X. Hypoxia-induced miR-214 expression promotes tumour cell proliferation and migration by enhancing the Warburg effect in gastric carcinoma cells. Cancer Lett. 2018; 414:44-56.

8. Yang Y, Li Z, Yuan H, Ji W, Wang K, Lu T, Yu Y, Zeng Q, Li F, Xia W, Lu S. Reciprocal regulatory mechanism between miR-214-3p and FGFR1 in FGFR1-amplified lung Oncogenesis. 2019;8(9):50.

9. Liao J, Lin J, Lin D, Zou C, Kurata J, Lin R, He Z, Su Down-regulation of miR-214 reverses erlotinib resistance in non-small-cell lung cancer through up-regulating LHX6 expression. Sci Rep. 2017;7(1):781. 
10. Zhang ZQ, Huang XM, Lu H. Early biomarkers as predictors for bronchopulmonary dysplasia in preterm infants: a systematic Eur J Pediatr. 2014;173(1):15-23.

11. Zhang ZQ, Zhong Y, Li XX, Huang XM, Du LZ. The effect of anti-placental growth factor antibody treatment on abnormal development of pulmonary vasculature and alveoli in hyperoxia-exposed neonatal rats. Braz J Med Biol Res. 2020; 53(2): e8917.

12. Bellik L, Vinci MC, Filippi S, Ledda F, Parenti A. Intracellular pathways triggered by the selective FLT-1agonist placental growth factor in vascular smooth muscle cells exposed to hypoxia. $\mathrm{Br} \mathrm{J}$ Pharmacol. 2005;146(4):568-75.

13. Chao CM, van den Bruck R, Lork S, Merkle J, Krampen L, Weil PP, Aydin M, Bellusci S, Jenke AC, Postberg J. Neonatal exposure to hyperoxia leads to persistent disturbances in pulmonary histone signatures associated with NOS3 and STAT3 in a mouse model. Clin Epigenetics. 2018; 10:37.

14. Cetinkaya M, Cansev M, Kafa IM, Tayman C, Cekmez F, Canpolat FE, Tunc T, Sarici SU. Cytidine 5'diphosphocholine ameliorates hyperoxic lung injury in a neonatal rat model. Pediatr Res. 2013;74(1):26-33.

15. de Visser YP, Walther FJ, Laghmani el H, Steendijk P, Middeldorp M, van der Laarse A, Wagenaar GT. Phosphodiesterase 4 inhibition attenuates persistent heart and lung injury by neonatal hyperoxia in rats. Am J Physiol Lung Cell Mol Physiol. 2012;302(1): L56-67.

16. Janer J, Andersson S, Haglund C, Karikoski R, Lassus P. Placental growth factor and vascular endothelial growth factor receptor-2 in human lung development. Pediatrics. 2008;122(2):340-6.

17. Chen X, Du J, Jiang R, Li L. MicroRNA-214 inhibits the proliferation and invasion of lung carcinoma cells by targeting JAK1. Am J Transl Res. 2018;10(4):1164-71.

18. Qi L, Jiang J, Jin P, Kuang M, Wei Q, Shi F, Mao D. Expression patterns of claudin-5 and its related signals during luteal regression in pseudopregnant rats: The enhanced effect of additional PGF treatment. Acta Histochem. 2018;120(3):221-7.

19. Rovani MT, Ilha GF, Gasperin BG, Nobrega JE, Jr., Siddappa D, Glanzner WG, Antoniazzi AQ, Bordignon V, Duggavathi R, Goncalves PBD. Prostaglandin F2alpha-induced luteolysis involves activation of Signal transducer and activator of transcription 3 and inhibition of AKT signaling in cattle. Mol Reprod Dev. 2017;84(6):486-94.

20. Principi N, Di Pietro GM, Esposito S. Bronchopulmonary dysplasia: clinical aspects and preventive and therapeutic strategies. J Transl Med. 2018;16(1):36.

21. Dravet-Gounot P, Torchin H, Goffinet F, Aubelle MS, El Ayoubi M, Lefevre C, Jarreau PH, Zana-Taieb E. Bronchopulmonary dysplasia in neonates born to mothers with preeclampsia: Impact of small for gestational age. PLoS One. 2018;13(9): e0204498.

22. Ogawa R, Mori R, lida K, Uchida Y, Oshiro M, Kageyama M, Kato Y, Tanaka T, Nakata Y, Nishimura Y, Hokuto I, Bonno M, Matsumoto N, Ito M, Takahashi N, Namba F. Effects of the early administration of sivelestat sodium on bronchopulmonary dysplasia in infants: A retrospective cohort study. Early Hum Dev. 2017; 115:71-6. 
23. Doyle LW, Cheong JL, Ehrenkranz RA, Halliday HL. Early (< 8 days) systemic postnatal corticosteroids for prevention of bronchopulmonary dysplasia in preterm infants. Cochrane Database Syst Rev. 2017;10:CD001146.

24. Sun Y, Kuek V, Liu Y, Tickner J, Yuan Y, Chen L, Zeng Z, Shao M, He W, Xu J. MiR-214 is an important regulator of the musculoskeletal metabolism and disease. J Cell Physiol. 2018;234(1):231-45.

25. Zhao Y, Ponnusamy M, Zhang L, Zhang Y, Liu C, Yu W, Wang K, Li P. The role of miR-214 in cardiovascular diseases. Eur J Pharmacol. 2017; 816:138-45.

26. Liu C, Luo J, Zhao YT, Wang ZY, Zhou J, Huang S, Huang JN, Long HX, Zhu B. TWIST1 upregulates miR-214 to promote epithelial-to-mesenchymal transition and metastasis in lung adenocarcinoma. Int J Mol Med. 2018;42(1):461-70.

27. Yang WC, Chen CY, Chou HC, Hsieh WS, Tsao PN. Angiogenic Factors in Cord Blood of Preterm Infants Predicts Subsequently Developing Bronchopulmonary Dysplasia. Pediatr Neonatol. 2015;56(6):382-5.

28. Gonsalves CS, Li C, Mpollo MS, Pullarkat V, Malik P, Tahara SM, Kalra VK. Erythropoietin- 1alpha and post-transcriptionally by miR-214 in sickle cell disease. Biochem J. 2015;468(3):409-23.

29. Chandrasekaran KS, Sathyanarayanan A, Karunagaran D. miR-214 activates TP53 but suppresses the expression of RELA, CTNNB1, and STAT3 in human cervical and colorectal cancer cells. Cell Biochem Funct. 2017;35(7):464-71.

30. Sitia R, Rubartelli A. The unconventional secretion of IL-1beta: Handling a dangerous weapon to optimize inflammatory responses. Semin Cell Dev Biol. 2018; 83:12-21.

31. Wang J, Bao L, Yu B, Liu Z, Han W, Deng C, Guo C. Interleukin-1beta Promotes Epithelial-Derived Alveolar Elastogenesis via alphavbeta6 Integrin-Dependent TGF-beta Cell Physiol Biochem. 2015;36(6):2198-216.

32. Cheng X, Shen Y, Li R. Targeting TNF: a therapeutic strategy for Alzheimer's disease. Drug Discov Today. 2014;19(11):1822-7.

33. Yue Y, Luo Z, Liao Z, Zhang L, Liu S, Wang M, Zhao F, Cao C, Ding Y, Yue S. Excessive activation of NMDA receptor inhibits the protective effect of endogenous bone marrow mesenchymal stem cells on promoting alveolarization in bronchopulmonary dysplasia. Am J Physiol Cell Physiol. 2019;316(6):C815-C27.

34. Ataie-Kachoie P, Pourgholami MH, Richardson DR, Morris DL. Gene of the month: Interleukin 6 (IL-6). J Clin Pathol. 2014;67(11):932-7.

35. Rocha G, Proenca E, Guedes A, Carvalho C, Areias A, Ramos JP, Rodrigues T, Guimaraes H. Cord blood levels of IL-6, IL-8 and IL-10 may be early predictors of bronchopulmonary dysplasia in preterm newborns small for gestational age. Dis Markers. 2012;33(1):51-60.

36. Newell LF, Holtan SG, Yates JE, Pereira L, Tyner JW, Burd I, Bagby GC. PIGF enhances TLR-dependent inflammatory responses in human mononuclear phagocytes. Am J Reprod Immunol. 2017;78(4).

37. van Mil A, Grundmann S, Goumans MJ, Lei Z, Oerlemans MI, Jaksani S, Doevendans PA, Sluijter JP. MicroRNA-214 inhibits angiogenesis by targeting Quaking and reducing angiogenic growth factor 
release. Cardiovasc Res. 2012;93(4):655-65.

38. Xu XH, Zhao C, Peng Q, Xie P, Liu QH. Kaempferol inhibited VEGF and PGF expression and in vitro angiogenesis of HRECs under diabetic-like environment. Braz J Med Biol Res. 2017;50(3): e5396.

\section{Figures}
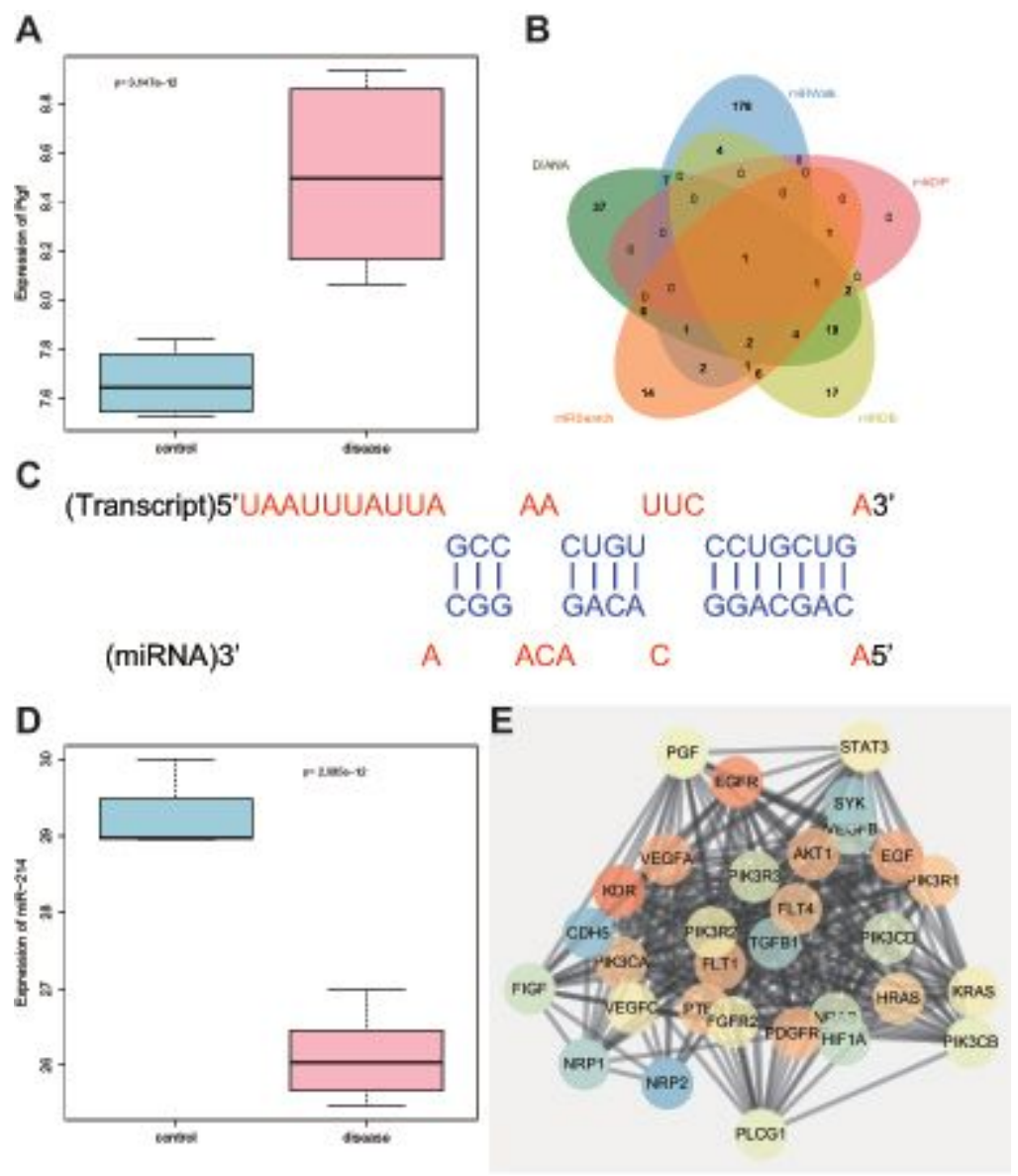

Figure 1

$\mathrm{PIGF} / \mathrm{miR}-214 / \mathrm{STAT} 3$ axis is predicted to be involved in BPD. A, PIGF expression in the dataset GSE25293-GPL1261, in which the blue box on the left indicates the expression of normal samples, and the red box on the right indicates the expression of BPD samples. B, The upstream miRNAs of PIGF predicted by DIANA Tools, miRWalk, mirDIP, miRDB and miRSearch databases. C, The binding site of PIGF and miR-214 predicted by DIANA Tools. D, miR-214 expression in the dataset GSE25293-GPL11199, in which the blue box on the left indicates the expression of normal samples, and the red box on the right indicates the expression of BPD samples. E, PPI analysis of PIGF (PGF), in which the redder gene sphere indicates greater importance, and the bluer gene sphere indicates a lower importance. 

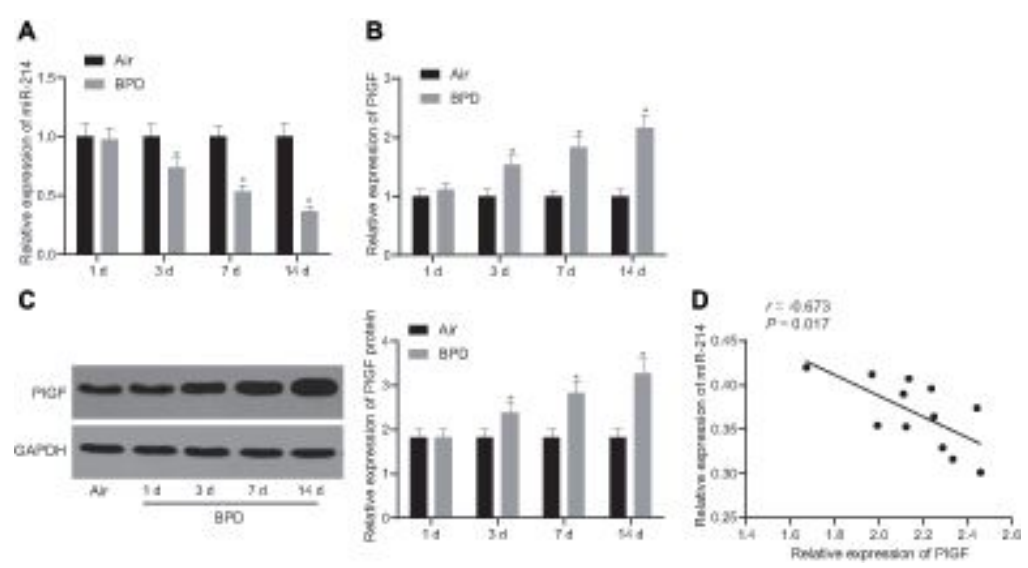

Figure 2

Decreased miR-214 expression and increased PIGF expression are found in the lung tissues of neonatal rats with BPD. A, miR-214 expression in neonatal rats with BPD detected by RT-qPCR normalized to U6. B, mRNA level of PIGF in neonatal rats with BPD detected by RT-qPCR normalized to GAPDH. C, Western blot analysis of PIGF protein expression in neonatal rats with BPD normalized to GAPDH. D, Correlation analysis of miR-214 and PIGF expression. The data were measurement data and expressed as mean \pm standard deviation. ${ }^{*} p<0.05$ vs. neonatal rats exposed to air. Data comparisons at different time points were performed by repeated measures ANOVA, followed by a Bonferroni post hoc test for multiple comparisons. $\mathrm{n}=12$.

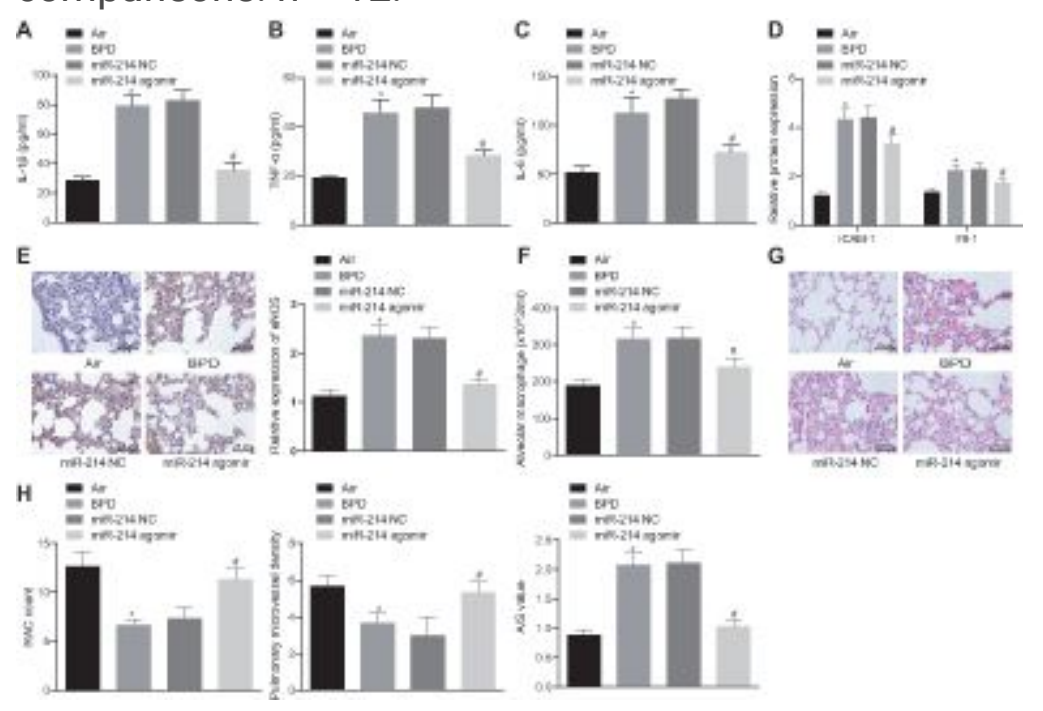

Figure 3

Overexpressed miR-214 represses pulmonary angiogenesis and alveolarization in neonatal rats with BPD. BPD neonatal rats were injected with miR-214 NC and miR-214 agomir. A-C, ELISA detection of the expression of inflammatory factors IL-1 $\beta$, TNF- $\otimes$ and IL- 6 in rats. D, Detection of ICAM- 1 and Flt- 1 levels in rats by ELISA. E, Immunohistochemistry of eNOS expression in pulmonary microvascular endothelium of rats $(\times 400)$. F, Giemsa staining of the number of macrophages in rats. G, HE staining of pulmonary microvascular $(\times 400)$. H, HE staining showing the number of alveoli, pulmonary microvessel count, and the growth of alveolar. The data were measurement data and expressed as mean \pm standard deviation. * 
$p<0.05$ vs. neonatal rats exposed to air. \# $p<0.05$ vs. BPD neonatal rats treated with miR-214 NC. Data comparisons among multiple groups were performed using one-way ANOVA and Tukey's for post hoc test, and $\mathrm{n}=12$.

A
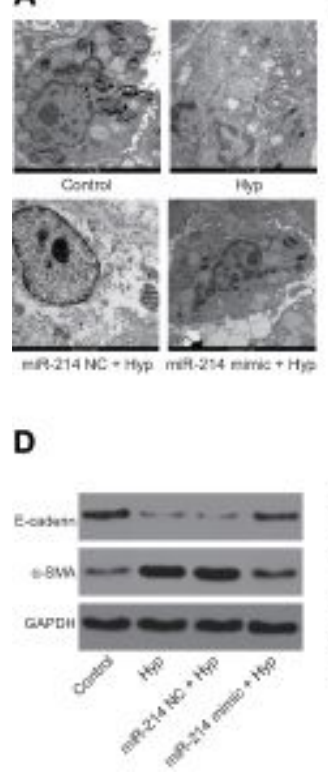

B
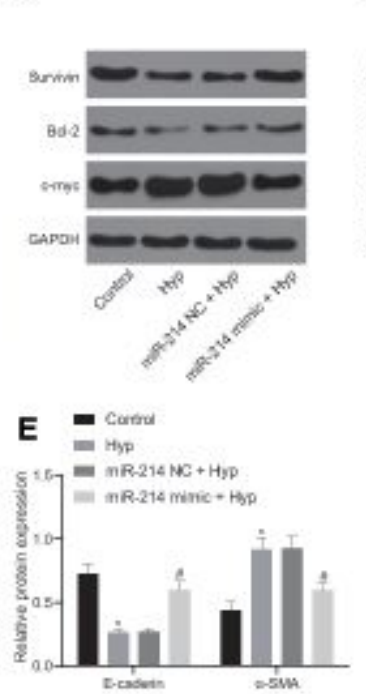

C

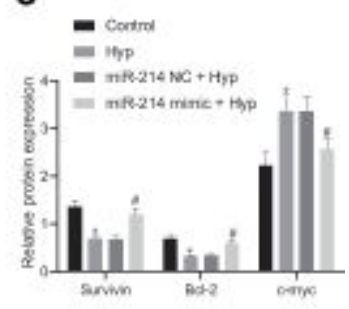

\section{Figure 4}

miR-214 overexpression exerts positive effects on the embryonic pulmonary epithelial cells of rats with BPD. Hyperoxic pulmonary epithelial cells were transfected with miR-214 NC or miR-214 mimic. A, The ultrastructure of alveolar epithelial cells under a transmission electron microscope (× 10000). B-C, Western blot analysis of Survivin, Bcl-2 and c-myc proteins in embryonic pulmonary epithelial cells. D-E, Western blot analysis of E-cadherin and a-SMA proteins in embryonic pulmonary epithelial cells. The data were measurement data and expressed as mean \pm standard deviation. ${ }^{*} p<0.05$ vs. pulmonary epithelial cells exposed to air. \# $p<0.05$ vs. hyperoxic pulmonary epithelial cells treated with miR-214 NC. Data comparisons were performed by one-way ANOVA, followed by a Tukey's post hoc test for multiple comparisons and the experiment was repeated three times.

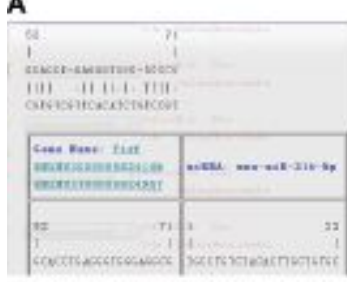

D

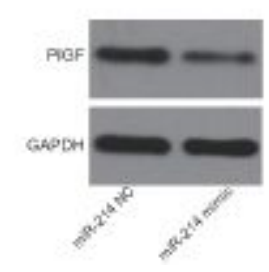

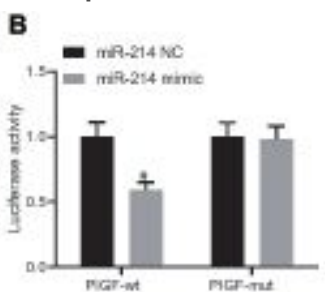

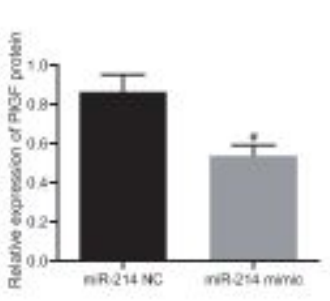

C
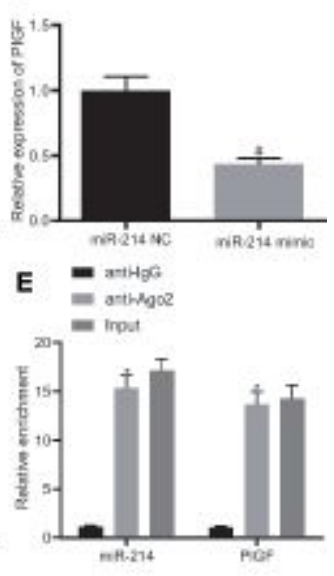

Figure 5 
PIGF is identified to be a target of miR-214. A, The predicted binding sites of miR-214 and PIGF. B, Dual luciferase reporter gene assay analysis of the binding of miR-214 to PIGF. C, RT-qPCR assay detection of mRNA level of PIGF after miR-214 overexpression. D, Western blot analysis of PIGF after miR-214 overexpression. E, RIP detection of the binding percentage of miR-214 and PIGF to Ago2 normalized to IgG binding. The data were measurement data and expressed as mean \pm standard deviation. ${ }^{*} p<0.05$ vs. anti-IgG. \# $p<0.05$ vs. cells transfected with miR-214 NC. An independent sample t-test was used for comparison between two groups. Data comparisons among multiple groups were performed by one-way ANOVA, followed by a Tukey's post hoc test for multiple comparisons. The experiment was repeated three times.

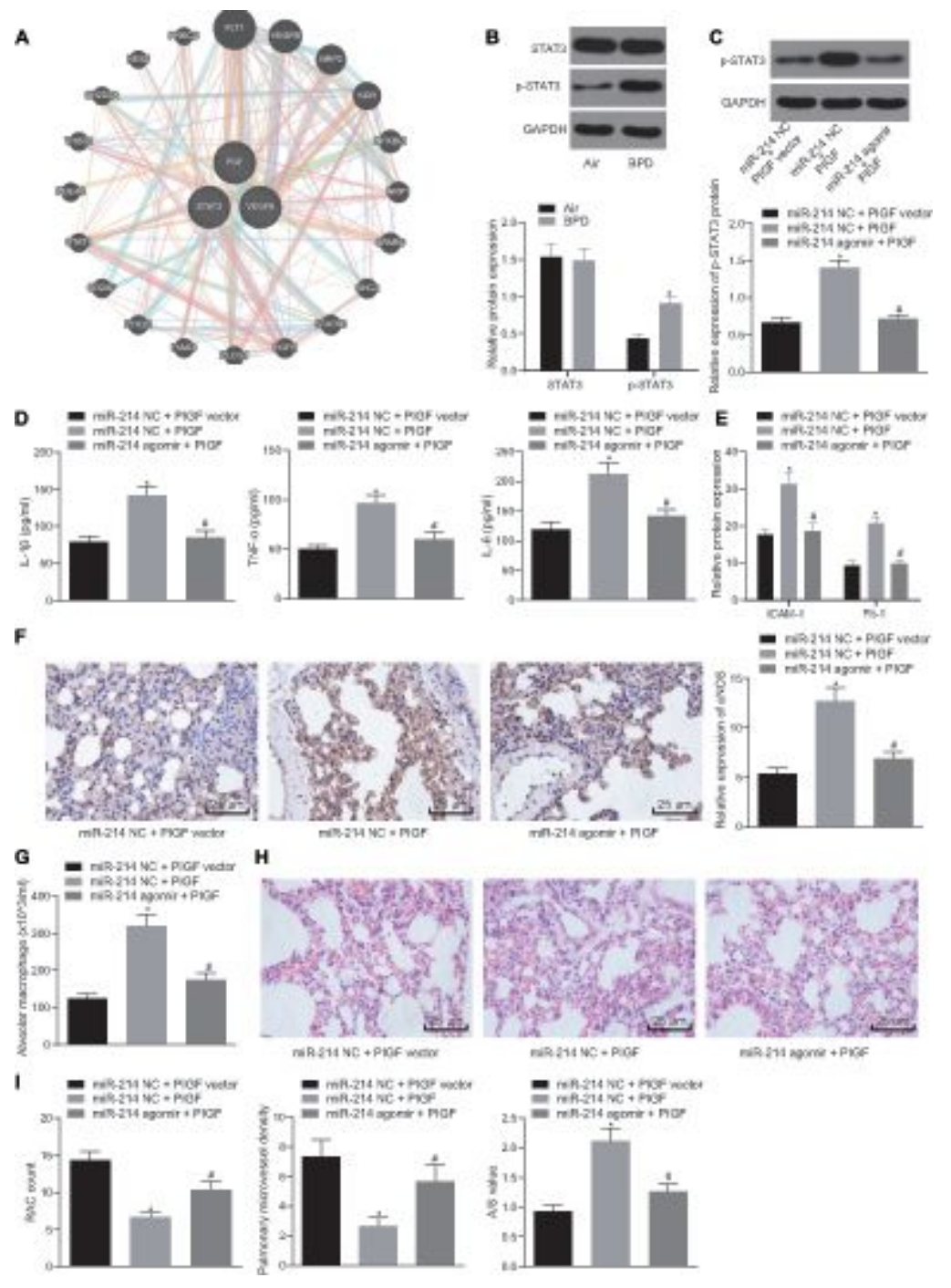

Figure 6

Overexpressed miR-214 blocked the effect of activated STAT3 signaling pathway on pulmonary angiogenesis and alveolarization by inhibiting PIGF in neonatal rats with BPD. A, The KEGG and GeneMANIA database were used to predict the downstream regulatory pathways of PIGF. B, Western blot analysis of the activation of STAT3 signaling pathway in BPD neonatal rats normalized to GAPDH. The neonatal rats with BPD were then respectively treated with miR-214 NC + PIGF vector, miR-214 NC + PIGF, or miR-214 agomir + PIGF. C, Western blot analysis of the expression of phosphorylated STAT3 in BPD 
neonatal rats normalized to GAPDH. D, Detection of the levels of inflammatory factors IL-1 $\beta, T N F-\square$ and IL- 6 by ELISA. E, Detection of levels of ICAM- 1 and FIt-1 by ELISA. F, Immunohistochemistry analysis of eNOS expression ( $\times 400)$. G, Giemsa staining detection of the number of macrophages. H, HE staining of the formation of pulmonary microvascular $(\times 400)$. I, HE staining of the number of alveoli and pulmonary microvessel, and alveolar growth. The data were measurement data and expressed as mean \pm standard deviation. ${ }^{*} p<0.05$ vs. BPD rats treated with miR-214 NC + PIGF NC. \# $p<0.05$ vs. BPD rats treated with miR-214 NC + PIGF. An independent sample t-test was used for comparison between two groups. Data comparisons among multiple groups were performed by one-way ANOVA, followed by a Tukey's post hoc test for multiple comparisons. $n=12$.
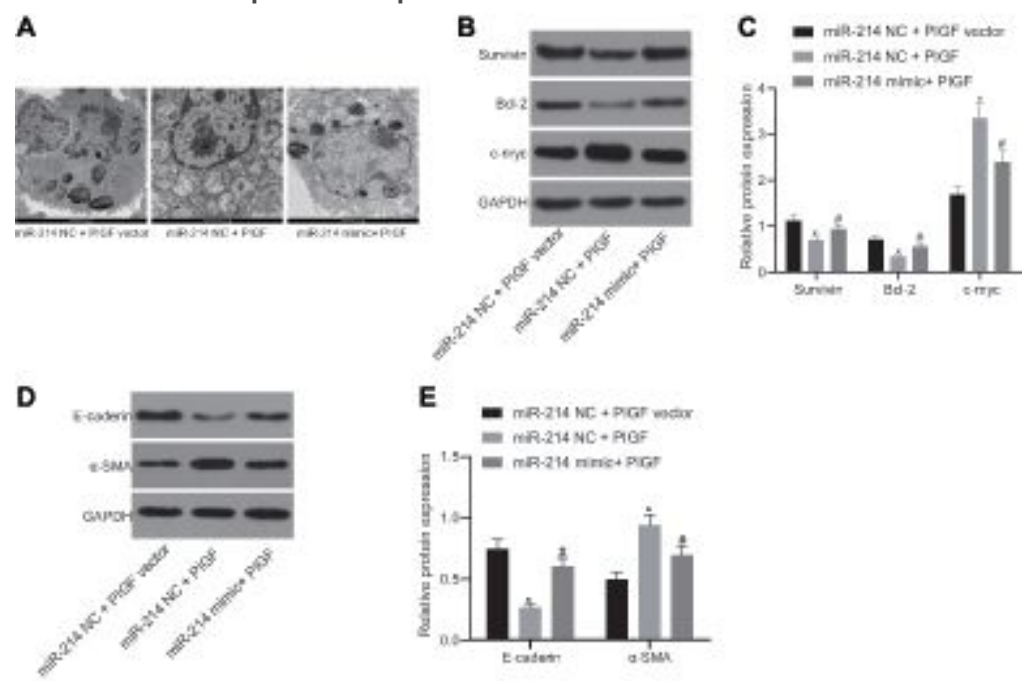

Figure 7

Overexpressed miR-214 blocked the effect of activated STAT3 signaling pathway on the bronchial embryonic pulmonary epithelial cells by inhibiting PIGF. A, The ultrastructure of alveolar epithelial cells under a transmission electron microscope $(\times 10000)$. B-C, Western blot analysis of the expressions of apoptotic factors Survivin, Bcl-2 and c-myc. D-E, Western blot analysis of the expression of E-cadherin and a-SMA in embryonic pulmonary epithelial cells. The data were measurement data and expressed as mean \pm standard deviation. ${ }^{*} p<0.05$ vs. the rats transfected with miR-214 NC and PIGF NC. \#p $<0.05$ vs. the rats transfected with miR-214 NC and PIGF. Data comparisons were performed by repeated measures ANOVA, followed by a Tukey's post hoc test for multiple comparisons. The experiment was repeated three times. STAT3, signal transducer and activator of transcription 3; PIGF, placental growth factor. 


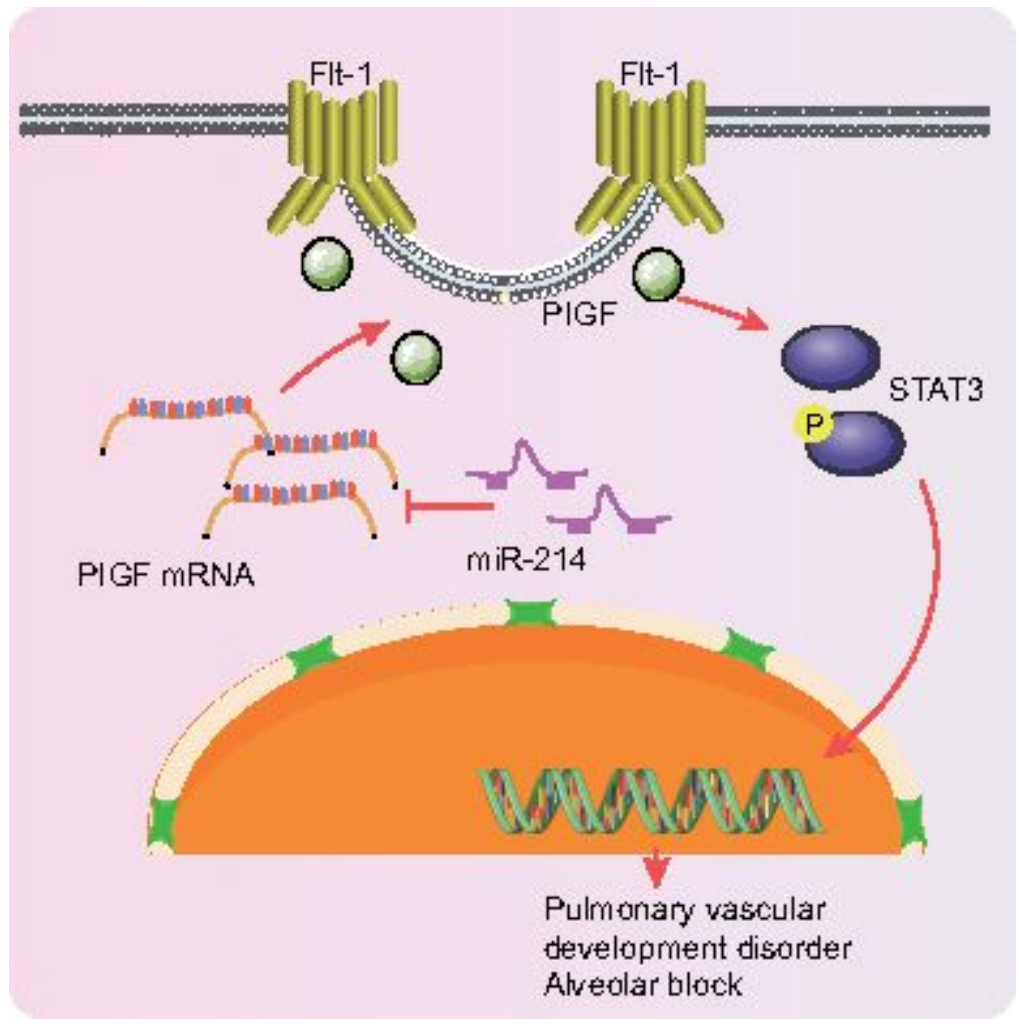

Figure 8

A schematic diagram illustrating the role of the miR-214-PIGF-STAT3 regulatory network in preterm infants with BPD. miR-214 can inhibit the activation of STAT3 signaling pathway by inhibiting the transcription of its downstream target gene PIGF, ultimately impeding pulmonary angiogenesis and alveolarization in preterm infants with BPD. BPD, bronchopulmonary dysplasia; STAT3, signal transducer and activator of transcription 3; PIGF, placental growth factor. 